doi.org/ 10.51891/rease.v7irr.3280

\title{
CARACTERIZAÇÃO DO PERFIL NUTRICIONAL DO REGISTRO DE INDIVÍDUOS COM EXCESSO DE PESO DA CIDADE DE BETIM - MG NO CONTEXTO DA PANDEMIA DE SARS-COV-2
}

\author{
CHARACTERIZATION OF THE NUTRITIONAL PROFILE OF THE REGISTRY OF \\ OVERWEIGHT INDIVIDUALS IN THE CITY OF BETIM - MG IN THE CONTEXT OF \\ THE SARS-COV-2 PANDEMIC
}

\author{
Leonardo Gonçalves Guimarães de Castro ${ }^{1}$ \\ Nathália Domingues Costa da Silva ${ }^{2}$ \\ Anne Josiele de Lima Vital ${ }^{3}$ \\ Raquel Silva Ferreira ${ }^{4}$ \\ Ingrid Werneck Linhares ${ }^{5}$
}

RESUMO: A vigilância alimentar e nutricional tem importância no contexto da promoção à saúde em diferentes esferas de governo, com o intuito de apoiar o desenvolvimento de um país. A partir dela é possível coletar, monitorar e avaliar informações, como uma forma de se obter parâmetros mais fidedignos para apoiar a tomada de decisão e intervenções em Alimentação e Nutrição. Contudo, ao longo do ano de 2020, do período pandêmico de Covid-ı, diante das novas recomendações que compreendem as dificuldades do cenário em questão, as ações em vigilância nutricional se mostram dificultadas. Neste sentido, tivemos como objetivo caracterizar os registros antropométricos no SISVAN (Sistema de Vigilância Alimentar e Nutricional), assim como dos diagnósticos nutricionais em município de médio porte da Região Metropolitana de Belo Horizonte em 2019 (pré-pandemia) e 2020 (período pandêmico Covid-I9). Foram emitidos relatórios disponíveis no site do SISVAN para o município de Betim. Os dados foram comparados por diagnóstico nutricional, faixa etária, utilizando como referência as médias entre os anos de 2019 e 2020. A análise estatística foi através do teste t de Student, com intervalo de confiança de $95 \%$ e $\mathrm{p}<0,05$. Por serem dados secundários não foi necessária submissão ao Comitê de Ética em Pesquisa (CEP). Neste estudo, demostramos que houve redução no registro de acompanhamento de crianças, adolescentes, adultos e gestantes com excesso de peso ( $p$ para registro de criança $=0,0 ; p$ para registro de adolescente $=0,00 ; p$ para registro de adulto $=0,00$; ( $p$ para registro de gestantes $=0,00$ ), exceto para idosos ( $p$ para registro de idoso $=0,20$ ) do que houve redução no índice de excesso de peso durante período pandêmico da Covid-19. Assim, nota-se que o impacto dos usuários ao serviço de saúde está atrelado ao período pandêmico, neste contexto, compreende-se que

\footnotetext{
${ }^{\mathrm{I}}$ Graduado em Biomedicina pela Pontifícia Universidade Católica de Minas Gerais - PUC Minas (2021). E-mail: lenardo7133@gmail.com

${ }^{2}$ Graduanda em Biomedicina pela Pontifícia Universidade Católica de Minas Gerais - PUC Minas.

${ }^{3}$ Graduanda em Biomedicina pela Pontifícia Universidade Católica de Minas Gerais - PUC Minas.

${ }^{4}$ Graduanda em Biomedicina pela Pontifícia Universidade Católica de Minas Gerais - PUC Minas.

${ }^{5}$ Doutoranda em Saúde da Criança e do Adolescente pela Universidade Federal de Minas Gerais - UFMG.

Mestre em Ciência de Alimentos pela UFMG (2012). Graduada em Nutrição pelo Centro Universitário

Newton Paiva (2002).E-mail: ingridwerneck@gmail.com
} 
a implementação de ações mais específicas se faz necessário diante do cenário exposto, visto sua importância no acompanhamento e registro desses usuários.

Palavras-chaves: Vigilância nutricional. Obesidade. Sars-Cov-2. Excesso de peso.

ABSTRACT: Food and nutrition surveillance is important in the context of health promotion in different spheres of government, with the aim of supporting the development of a country. From it, it is possible to collect, monitor and evaluate information, as a way to collect more reliable parameters to support the taking and functions in Food and Nutrition. However, throughout 2020, during the Covid-I9 pandemic period, in view of the new recommendations that understand the difficulties of the scenario in question, actions in nutritional surveillance are hampered. To characterize the anthropometric records in SISVAN (Food and Nutritional Surveillance System), as well as nutritional diagnoses in a medium-sized municipality in the Metropolitan Region of Belo Horizonte in 2019 (pre-pandemic) and 2020 (Covid-19 pandemic period). Reports available on the SISVAN website were issued for the municipality of Betim. Data were compared by nutritional diagnosis, age group, using as reference the means between the years 2019 and 2020. As they are secondary data, submission to the Research Ethics Committee (CEP) was not necessary. The distancing measures adopted to control the pandemic in 2020 impacted the access of users to the surveillance health service and, consequently, the recording of anthropometric data on nutritional status (body weight and measurements). Thus, a reduction in the monitoring record of overweight children, adolescentes, adults and pregnant ( $p$ for child record $=0.00 ; p$ for adolescent record $=0.00 ; p$ for adult record $=0.00$; ( $p$ for pregnant record=o,oo), except for elderly ( $p$ for elderly record $=0.20$ ) that there was a reduction in the weight index during the Covid-I9 pandemic period. Thus, it is noted that the impact of users on the health service is linked to the pandemic period, in this context, it is understood thvat the implementation of more specific actions is necessary in the above scenario, given its importance in monitoring and recording users.

Keywords: Nutritional Surveillance. Obesity. Sars-Cov-2. Overweight.

\section{INTRODUÇÃO}

O Sistema de Vigilância Alimentar e Nutricional (SISVAN) foi estabelecido no Brasil no ano de 1990, após deliberação da Lei 8080/1990 e a publicação da Portaria no 1156 de 31 de agosto do 1990, com o intuito de monitorar a situação nutricional e da alimentação da população, contribuindo, portanto, para a promoção da saúde e o desenvolvimento do país. A partir deste é possível coletar, monitorar e avaliar dados antropométricos e de consumo alimentar a fim de se obter parâmetros mais fidedignos sobre a alimentação, nutrição e saúde e os padrões de consumo alimentar da população (BRASIL, 2015).

O uso da antropometria, que consiste na ciência de avaliação das dimensões físicas do corpo humano em sua totalidade por meio de índices, como peso, idade, estatura e 
índice de massa corporal (MCCONVILLE et al, 1980), é de extrema relevância para a avaliação do estado nutricional e monitoramento de indivíduos de diferentes faixas etárias, principalmente com distúrbios do peso (HO, 200I; EJTAHED, 2018; OHARA et al, 2019; $\mathrm{ZHU}$ et al, 2020). Além disso, esses parâmetros atuam como um bom preditor de saúde e qualidade de vida da população (MONREAL et al., 2015).

A obesidade serve como porta de entrada para doenças de caráter multifatorial, como diabetes mellitus, hipertensão, dislipidemias, doença arterial coronariana e alguns tipos de câncer (SOLOMON, 1977; KNIGHT, 20II; MARTINS, 2018). De acordo com a Organização Mundial de Saúde (OMS) estima que mais de 2 bilhões de pessoas estejam com sobrepeso ou obesidade em todo o mundo. No Brasil, cerca de $60 \%$ a $65 \%$ da população apresenta excesso de peso corporal (BRASIL, 2020), bem como o excesso de peso, a baixa estatura e magreza podem trazer prejuízos à saúde, principalmente, em crianças e adolescentes com doença falciforme (DEWEY et al, 2oır; SOUZA et al, 20II).

Os distúrbios psíquicos influenciam nos hábitos alimentares dos pacientes podendo causar distúrbios tais como compulsão alimentar e obesidade (ZWAAN, 20or; AGUERA, 2021). No Brasil, a depressão e os distúrbios relacionados à ansiedade atingem cerca de I8,6 milhões de brasileiros. No período pandêmico da Covid-ı́ em função das novas medidas para contenção do vírus, como isolamento social, os indivíduos ficaram mais expostos a possíveis impactos na saúde de origem psíquica (MALTA et al., 2020).

Considerando os possíveis impactos à saúde alimentar e nutricional da população em relação ao período pandêmico de Covid-ı9, a necessidade de medidas de acompanhamento e monitoramento nutricional foram reforçadas. Contudo, diante das novas recomendações que compreendem as dificuldades deste cenário ao longo do ano de 2020, as ações em vigilância nutricional foram dificultadas (DUTRA, et al., 2020).

Neste contexto, o estudo teve como objetivo caracterizar os registros antropométricos no SISVAN de indivíduos de diferente faixa etárias que frequentam as Unidades Básicas de Saúde UBS do Sistema Único de Saúde SUS, bem como dos diagnósticos nutricionais na cidade de Betim, região metropolitana do estado de Minas Gerais em 2019 pré-pandemia e 2020 período pandêmico de Covid-ı. 


\section{MÉTODOS}

Trata-se de um estudo retrospectivo desenvolvido dentre um período compreendido de junho a novembro de 2021 a partir da análise do banco de dados do Sistema de Vigilância Alimentar e Nutricional SISVAN (https://sisaps.saude.gov.br/sisvan/), referente aos dados antropométricos de excesso de peso de usuários das UBSs do município de Betim nos anos de 2019 (período pré-pandêmico) e 2020 (período pandêmico) de Covid-19. De acordo com os Censos Demográficos de 2019 e de 2020 do Instituto Brasileiro de Geografia e Estatística - IBGE (https://www.ibge.gov.br/) da população brasileira, a cidade de Betim, no ano de 2019, apresentava 439.340 habitantes e, no ano de 2020, apresentava 444.784 habitantes.

Os dados foram compilados em planilhas e quadros e comparados de acordo com o ano de referência, faixa etária, região, número absoluto e percentuais. Os dados foram analisados por faixa etárias criança, adolescente, adulto, gestante e idoso com base nos dados antropométricos peso e altura. $\mathrm{O}$ excesso de peso foi compreendido, neste estudo, pela soma de sobrepeso, obesidade e obesidade mórbida.

A análise foi realizada usando de referência as médias dos grupos e as comparações

entre os anos estudados. Para comparação das médias das variáveis quantitativas foi utilizado o teste $t$ de Student com intervalo de confiança de $95 \%$ e p<0,05 através do pacote de recurso Real Statistics.

Esta pesquisa não necessitou de aprovação em Comitê de Ética em Pesquisa, por serem dados consolidados, e públicos dos bancos de dados oficiais do ministério da saúde.

\section{RESULTADOS E DISCUSSÃO}

Este estudo analisou os registros de diagnóstico nutricional de excesso de peso de indivíduos de diferentes faixas etárias da cidade de Betim disponíveis no SISVAN durante o período pré-pandêmico do ano de 2019 e o período pandêmico de Covid-ı́ do ano de 2020 .

Tabela r. Dados absolutos da população e cobertura nutricional do município de Betim

$\begin{array}{lll} & 2019 & 2020 \\ \text { População de Betim } & 439.340 & 444.784 \\ \text { Usuários Sisvan Web } & 38.702 & 13.568\end{array}$


Usuários Sisvan Web com excesso de

I6.973

8.324

peso

Fonte: Fonte: SISVAN Web 2021; IBGE, 2021

Como representado na Tabela I, no período pré-pandêmico de Covid-ı́ do ano de 2019, na cidade de Betim, haviam registrados 439.340 habitantes, sendo 38.702 cadastros antropométricos no SISVAN, que representa $8 \%$ da população do município, sendo 3\% com excesso de peso. Já no período pandêmico de Covid-19 do ano de 2020, esta cidade apresentava 444.784 habitantes, sendo 13.568 cadastros antropométricos registrados, que representa $3 \%$ da população, sendo $2 \%$ com excesso de peso. Demostramos que a cobertura populacional do SISVAN da cidade de Betim foi de somente $3 \%$ no ano de 2020 em relação aos $8 \%$ correspondentes ao ano de 2019. Houve redução também para o registro de indivíduos com excesso de peso, que neste período representou somente $2 \%$ da população. $O$ monitoramento nutricional se mostrou eficaz para redução do risco cardiovascular em indivíduos com excesso de peso (ALVAREZ et al., 2009). Além disso, indivíduos com excesso submetidos a maior acompanhamento nutricional demostraram redução de distúrbios emocionais (POÍNHOS et al., 2004).

Tabela 2. Dados absolutos de usuários com excesso de peso conforme a faixa etária registrados nas UBS da cidade de Betim para comparação de médias obtidas entre o ano 2019 (Período pré-pandêmico) e o ano de 2020 (período pandêmico) de Covid-ı.

\section{UBS}

Ano
Alcides Braz
Amazonas
Citrolândia
Larajeiras
Alterosas
Imbiruçu
CAIC
Campos Eliseos
PC
Bemge
Cruzeiro do Sul
Cidade Verde
Petrovale
DC

\section{Faixas etárias}

Criança Adolescent Adulto Gestante Idoso
e

$\begin{array}{cccccccccc}2019 & 2020 & 2019 & 2020 & 2019 & 2020 & 2019 & 2020 & 2019 & 2020 \\ 37 & 4 & 232 & 56 & 85 & 13 & 21 & 34 & 16 & 5 \\ 6 & 3 & 35 & 30 & 19 & 4 & 4 & 6 & 2 & 4 \\ 80 & 9 & 381 & 41 & 151 & 7 & 14 & 6 & 17 & 5 \\ 39 & 9 & 307 & 135 & 89 & 10 & 8 & 38 & 31 & 22 \\ 38 & 12 & 201 & 97 & 100 & 9 & 13 & 30 & \text { II } & 20 \\ 47 & 13 & 287 & 82 & 89 & 3 & 13 & 55 & 17 & \text { I } \\ 58 & 13 & 328 & 90 & 121 & 7 & 23 & 64 & 15 & 0 \\ 56 & 18 & 311 & 185 & 93 & 23 & 24 & 49 & 10 & 13 \\ 80 & 36 & 356 & 200 & 88 & 49 & 9 & 31 & 15 & 17 \\ 52 & 12 & 369 & 72 & 160 & 15 & 15 & 28 & 39 & 10 \\ 16 & 21 & 102 & 140 & 74 & 5 & 17 & 75 & 0 & 6 \\ 6 & 0 & 51 & 43 & 15 & \text { II } & 3 & 17 & \text { I } & 2 \\ 56 & 29 & 343 & 192 & 85 & 29 & 4 & 26 & \text { II } & 3 \\ 27 & 44 & 252 & 367 & 60 & \text { IIO } & \text { I } & 29 & 6 & \text { II }\end{array}$




\begin{tabular}{|c|c|c|c|c|c|c|c|c|c|c|}
\hline Vila Recreio & IO & 2 & 88 & 29 & 46 & 5 & 7 & 2 & 2 & I \\
\hline PTB & 29 & 9 & 195 & 9I & II6 & 6 & 5 & I9 & 38 & 36 \\
\hline Guanabara & 59 & 4 & 182 & 12 & 71 & 2 & 4 & 7 & 3 & o \\
\hline Homero Gil & 95 & 6 & 551 & 102 & 172 & I & $3 I$ & 49 & $2 \mathrm{I}$ & 3 \\
\hline Icaivera & 48 & 5 & 209 & $2 \mathrm{I}$ & 59 & 9 & 5 & o & 7 & 3 \\
\hline Alvorada & 21 & 2 & 132 & 13 & 55 & 2 & 4 & 5 & 4 & 6 \\
\hline Universal & I7 & 4 & 48 & 25 & 44 & 19 & 3 & o & 4 & 5 \\
\hline Bueno Franco & 46 & II & 317 & I66 & II 8 & 28 & II & 56 & 7 & 6 \\
\hline Marimba & 34 & 4 & 156 & 45 & 52 & 3 & 26 & 34 & 4 & o \\
\hline Colônia & 77 & 9 & 372 & 49 & 96 & 3 & IO & 38 & 12 & o \\
\hline Trincheira & 74 & I & 422 & 36 & 95 & 4 & 7 & 24 & IO & o \\
\hline NSF & $6 \mathrm{I}$ & 30 & I73 & I86 & $7 \mathrm{I}$ & 43 & 2 & 20 & 5 & 33 \\
\hline Vianópolis & 15 & 5 & 63 & 43 & 29 & 3 & 18 & 27 & o & o \\
\hline Paulo Camilo & 44 & 22 & 202 & 156 & 93 & 45 & II & 25 & I & o \\
\hline Angola & 58 & 8 & 266 & 62 & I04 & 12 & 5 & 28 & 7 & I \\
\hline Petrópolis & I6o & 7 & 618 & 70 & 226 & 23 & I5 & $\mathrm{I} 4$ & 39 & 5 \\
\hline Bandeirinhas & 37 & 12 & 269 & I47 & $8 \mathrm{I}$ & IO & I2 & 40 & 22 & 29 \\
\hline Dom Bosco & 54 & 3 & 295 & 60 & 92 & 3 & 9 & 34 & 9 & I \\
\hline Teresópolis & 30 & 9 & I76 & 93 & 66 & I5 & I2 & $3 \mathrm{I}$ & $\mathrm{I} 4$ & 8 \\
\hline Vila Cristina & $7 \mathrm{I}$ & 43 & 442 & 275 & II 2 & 95 & IO & 7 & I9 & 8 \\
\hline Nova Baden & $4 \mathrm{I}$ & 20 & 296 & 295 & 83 & 20 & 4 & 30 & 54 & 68 \\
\hline Cachoeira & 45 & 24 & 220 & I5I & 220 & I $5 \mathrm{I}$ & 3 & 13 & I7 & I5 \\
\hline Total (n) & 1724 & 463 & 9247 & 3857 & 5129 & 2666 & 383 & 991 & 490 & 347 \\
\hline
\end{tabular}

Tabela 3. Medidas de média, desvio-padrão e teste $t$-Student de usuários com excesso de peso conforme a faixa etária atendidos nas UBS da cidade de Betim entre o ano 2019 (Período pré-pandêmico) e o ano de 2020 (período pandêmico) de Covid-ı.

\begin{tabular}{|c|c|c|c|c|c|c|c|}
\hline \multicolumn{3}{|c|}{2019} & \multicolumn{2}{|c|}{2020} & & & \\
\hline Faixa etária & Média & $\mathrm{DP}$ & Média & $\mathrm{DP}$ & $\mathrm{T}$ & $\mathrm{gl}$ & p-valor \\
\hline Criança & 87,77 & 42,51 & 19,25 & 24,62 & 8,36 & 56 & 0,00 \\
\hline Adolescente & 47,88 & 29,33 & 12,86 & 11,56 & 6,66 & 46 & 0,00 \\
\hline Adulto & 256,86 & I35, 19 & 107,13 & 84,57 & 5,63 & 59 & 0,00 \\
\hline Gestante & 10,63 & 7,45 & 27,52 & 18,29 & 5,12 & 46 & 0,00 \\
\hline Idoso & 13,61 & 12,75 & 9,63 & I3.8I & $\mathrm{I}, 26$ & 70 & 0,20 \\
\hline
\end{tabular}

Fonte: Elaborado pelos autores

As medidas de distanciamento adotadas, que tiveram como intuito o controle da pandemia no ano de 2020, impactaram na assistência à saúde ocasionando a redução dos dados coletados para vigilância nutricional. Assim, houve redução no registro do acompanhamento de crianças, adolescentes e adultos com excesso de peso ( $p$ para registro de criança $=0,0 ; p$ para registro de adolescente $=0,00 ; p$ para registro de adulto $=0,00 ; p$ para 
registro de gestante $=0,00$ ), exceto para idosos ( $p$ para registro de idoso $=0,20$ ) do que houve redução no índice de excesso de peso durante o período pandêmico em questão.

Demonstramos redução no número de registros de gestantes ( $\mathrm{p}$ para registro de gestantes=0,0o) no período pandêmico da Covid-I9. Os municípios do estado do Rio Grande do Sul, também demonstraram efeito semelhante, sendo as gestantes os usuários mais afetados neste período (STEFFLER et al., 2021). A redução do monitoramento nutricional destes usuários agrava o cenário pandêmico, já que indivíduos com excesso de peso expostos ao Sars-Cov-2 são mais propensos a manifestar quadros mais graves da doença, necessitar de unidade de terapia intensiva (UTI) e evoluir para óbito (CAI et al., 202I). Além disso, neste mesmo período, demonstramos redução significativa para os registros de crianças ( $p$ para registro de criança=o,o), adolescentes ( $p$ para registro de adolescente $=0,00$ ) e adultos ( $p$ para registro de adulto=o,oo). Assim como no nosso resultado, o estado do nordeste demonstrou baixa cobertura nutricional do que foi inferior a 50\% para crianças (PESSOA et al., 202I).

\section{CONCLUSÃO}

Neste estudo, entendemos que o impacto da cobertura nutricional está atrelado ao período pandêmico, e que o monitoramento nutricional de usuários de diferentes faixas etárias, principalmente, crianças, adolescentes, adultos e gestantes foram os mais afetados neste período, neste contexto, compreende-se que a implementação de ações mais específicas se faz necessário diante do cenário exposto, visto sua importância no acompanhamento e registro desses usuários. O estudo se limitou a um município, por isso é importante que novos estudos sejam realizados considerando outros municípios.

\section{REFERÊNCIAS}

AGUERA, Z.; MADRID M. L.; BAGUÉ, N. M. et al. review of binge eating disorder and obesity. Neuropsychiatrie, v. 35, p. 57-67, 2021. DOI: https://doi.org/10.1007/s4021I-020o0346-w. Disponível em: https://link.springer.com/article/10.1007/s402II-020-00346W\#citeas. Acesso em: or dez. 2021.

ALVAREZ, T. S.; ZANELLA, M. T. Impacto de dois programas de educação nutricional sobre o risco cardiovascular em pacientes hipertensos e com excesso de peso. Revista 
nutrição, v. 22, n. I, p. 71-79, 2009. Disponível em: https://www.scielo.br/j/rn/a/n3Qi7WDGv93 NRdzWmnjpFNg/abstract/?lang=pt. Acesso em: 19 out. 2021.

BRASIL. (202I). Ministério da Saúde. Protocolos do Sistema de Vigilância Alimentar e Nutricional SISVAN na assistência à saúde. BRASIL. Disponível em: https://aps.saude.gov.br/biblioteca/visualizar/MTI4 $\mathrm{Ng}==$. Acesso em: 02 nov. 202I.

BRASIL (202I). Ministério da Saúde. Secretaria de Atenção à Saúde. Departamento de Atenção Básica. Marco de referência da vigilância alimentar e nutricional na atenção básica. Brasil.

Disponível em: http://bvsms.saude.gov.br/bvs/publicacoes/marco_referencia_vigilancia_alimentar.pdf. Acesso em: 12 ago. 2021.

BRASIL (2020). Ministério da Saúde. Secretaria de Vigilância em Saúde. Vigitel Brasil: vigilância de fatores de risco e proteção para doenças crônicas por inquérito telefônico. Brasil. Disponível em: https://bvsms.saude.gov.br/bvs/publicacoes/vigitel_brasil_2019_vigilancia_fatores_risco.p df. Acesso em: I2 ago. 202I.

CAI, Z.; YANG, Y.; ZHANG, J. Obesity is associated with severe disease and mortality in patients with coronavirus disease 2019 (COVID-19): a meta-analysis. BMC Public Health, v. 2I, n. I522, I-I4, 202I. Disponível em: https://bmcpublichealth.biomedcentral.com/track/pdf/Io.II86/sI2889-02I-II546-6.pdf. Acesso em: II set. 202I.

DEWEY, K. G.; BEGUM, D. K. Long-term consequences of stunting in early life. Maternal \& Child Nutrition, v. 7, n. 3, p. 5-18, 2021. Disponível em: https://pubmed.ncbi.nlm.nih.gov/21929633/. Acesso em: or dez. 202I.

DUTRA, A. F. O. et al. THE IMPORTANCE OF HEALTHY EATING AND ADEQUATE NUTRITIONAL STATUS IN THE FACE OF THE COVID-i9 PANDEMIC. Brazilian Journal of Development, v. 6, n. 9. p. I-Io, 2020. DOI I0.34117/bjdv6n9-I8I. em: https://www.researchgate.net/publication/346078453_A_IMPORTANCIA_DA_ALIME NTACAO_SAUDAVEL_E_ESTADO_NUTRICIONAL_ADEQUADO_FRENTE_A_ PANDEMIA_DE_COVID- 
I9_THE_IMPORTANCE_OF_HEALTHY_EATING_AND_ADEQUATE_NUTRITIO NAL_STATUS_IN_THE_FACE_OF_THE_COVID-i9_PANDEMIC. Acesso em: 7 out. 2021.

EJTAHED, H. S.; QORBANI, M.; MOTLAGH, M. E.; et al. Association of anthropometric indices with continuous metabolic syndrome in children and adolescents: the CASPIAN-V study. Eating and Weight Disorders - Studies on Anorexia, Bulimia and Obesity, v. 23, n. 5, p 597-604, 2018. Disponivel em: https://pubmed.ncbi.nlm.nih.gov/29094277/. Acesso em: II set. 2021.

HO, S. C.; CHEN, Y. M.; WOO, J. L. F. et al., Association between simple anthropometric indices and cardiovascular risk factors. (200I). International Journal of Obesity. v. 25, n. p. II, I689-1697, 200I. DOI https://doi.org/10.1038/sj.ijo.0801784. Disponível em: https://www.nature.com/articles/o8or784\#citeas. Acesso em: II set. 202I. IBGE. Instituto Brasileiro de Geografia e Estatística. Censos Demográficos de Betim de 2019 e de 2020. 2021. Disponível em: https://cidades.ibge.gov.br/brasil/mg/betim. Acesso em: IO ago. 202I.

KNIGHT, J. A. Diseases and Disorders Associated with Excess Body Weight. Ann Clin Lab Sci Spring, v 4I, n. 2. p. I07-I2, 201I. Disponível em: http://www.annclinlabsci.org/content/4I/2/107.short. Acesso em: or dez. 2021.

MALTA, D. C.; SILVA, D. R. P. Distanciamento social, sentimento de tristeza e estilos de vida da população brasileira durante a pandemia de Covid-ı. Saúde debate. V. 44, n. 4. P. 2020. I77-190, Disponível em: https://www.scielosp.org/article/sdeb/2020.v44nspe4/177-190/

MARTINS, A. P. B. É PRECISO TRATAR A OBESIDADE COMO UM PROBLEMA DE SAÚDE PÚBLICA. (2018). Revista de Administração de Empresas. v. 58, n. 3, p. 33734I, 2018. Disponível em: https://www.scielo.br/j/rae/a/RLQv6c8QghbDdXCt4hSxkhG/?lang=pt. Acesso em: or dez. 2021.

MCCONVille, J. T.; ClAuser, C. E.; CHURCHIll, T. D.; et al. Anthropometric Relationships of Body and Body Segment Moments of Inertia. TEXAS INSTITUTE FOR REHABILITATION AND RESEARCH, p. I-II3, I980 Disponível em: https://apps.dtic.mil/sti/citations/ADA097238 
MONREAL, A.M.J.; MURCIA, M.; MURCIA, V. G.; et al. Anthropometric and Quality-of-Life Parameters in Acute Intermittent Porphyria Patients. Medicine, v. 94, n. 30. p. $\mathrm{I}-8, \quad$ 2015. Disponível em: https://www.ncbi.nlm.nih.gov/pmc/articles/PMC4554130/. Acesso em: or dez. 202I. OHARA, K.; MASE, T.; KOUDA, K. et al. Association of anthropometric status, perceived stress, and personality traits with eating behavior in university students. (2019) Eating and Weight Disorders - Studies on Anorexia, Bulimia and Obesity, v. 24, n. 3, p. 521-531, 2019. Disponível em: https://link.springer.com/article/I0.1007/s40519-018-0o637-w. Acesso em: or dez. 2021.

OMS. Organização Mundial da Saúde (2020). Organização das Nações Unidas (ONU). Depressão é tema de campanha da OMS para Dia Mundial da Saúde de 2017. Disponível em: https://nacoesunidas.org/depressao-e-tema-de-campanha-da-oms-para-diamundialda-saude-de-2017/. Acesso em: or nov. 2021.

OMS. Organização Mundial da Saúde (2020). Organização das Nações Unidas (ONU). OMS registra aumento de casos de depressão em todo o mundo; no Brasil são II,5 milhões de pessoas Disponível em: https://nacoesunidas.org/oms-registra-aumento-de-casos-dedepressao-emtodo-o-mundo-no-brasil-sao-II5-milhoes-de-pessoas/. Acesso em: or nov. 2021.

PESSOA, J. T. et al. Vigilância alimentar e nutricional: Cobertura e caracterização para crianças menores de 2 anos do Nordeste brasileiro. Research, Society and Development, v. Io, n.5, p. $\quad$ I-I4, 202I. $\quad$ Disponível em: https://rsdjournal.org/index.php/rsd/article/view/r4909/13363. Acesso em: or nov. 2021. POÍNHOS, R. M. A. Efeitos da motivação e do acompanhamento no tratamento da obesidade. Orientador: Dra. Flora Correia. 2004. 96 p. Tese (Licenciatura em Ciências da Nutrição) - Faculdade de Ciências da Nutrição e Alimentação da Universidade do Porto, FCNAUP, 2004. Disponível em: https://repositorio-aberto.up.pt/handle/I0216/54649. Acesso em: 24 set. 202I.

STEFFLER, B.; LEAL, G. V. S.; KIRSTRN, V. R. IMPACTOS DA PANDEMIA DO COVID-I9 NA COBERTURA DA VIGILÂNCIA ALIMENTAR E NUTRICIONAL: UMA ANÁLISE DOS DADOS DE ESTADO NUTRICIONAL NOS MUNICÍPIOS DO RIO GRANDE DO SUL. Congresso Internacional em Saúde. Resumo simples. 
Nutrição,

n. 8 ,

p.

$\mathrm{I}-2$,

2021.

https://publicacoeseventos.unijui.edu.br/index.php/conintsau/article/view/19507. Acesso em: or dez. 2021.

SOLOMON, C.G., MANSON, J.E. Obesity and mortality: a review of the epidemiologic. The American Journal of Clinical Nutrition. v. 66, n 4, p. I444-1050, 1977. Disponível em: https://academic.oup.com/ajen/article/66/4/1044S/4656org?login=true. Acesso em: or dez. 202I.

SOUZA, K. C. M.; ARAUJO, P. I. C.; SOUZA-JUNIOR, P. R.; et al. Baixa estatura e magreza em crianças e adolescentes com doença falciforme. Rev. Nutr, v, 24, n. 6, p. 853826, 2021. Disponível em: https://www.scielo.br/j/rn/a/rPJwWFkmZBWY ${ }_{4} \mathrm{Hf}_{4} \mathrm{~h}_{s N X n n j} /$ ?lang=pt. Acesso em: 02 nov. 2021.

ZHU, X, L.; CHEN, Z. H.; LI, Y. et al. Associations of vitamin D with novel and traditional anthropometric indices according to age and sex: a cross-sectional study in central southern China. Eating and Weight Disorders - Studies on Anorexia, Bulimia and Obesity, v. 25. P. I65I-I66I, 2020 Disponivel em https://link.springer.com/article/ı.1007/s40519-019-00803-8 ZWAAN, M. Binge eating disorder and obesity. International Journal of Obesity, v 25, p. 51-55, 2001. DOI: https://doi.org/ro.1038/sj.ijo.o8or699. Disponível em: https://www.nature.com/articles/o8or699\#citeas. Acesso em: or dez. 2021. 